\title{
Molecularly imprinted solid-phase extraction combined with high performance liquid chromatography for analysis of phenolic compounds from environmental water samples
}

\author{
Qin-Zhong Feng ${ }^{\mathrm{a}}$, Li-Xia Zhao ${ }^{\mathrm{a}, *}$, Wei Yan ${ }^{\mathrm{a}}$, Jin-Ming Lin ${ }^{\mathrm{a}, \mathrm{b}, *}$, Zhi-Xia Zheng $^{\mathrm{c}}$ \\ a State Key Laboratory of Environmental Chemistry and Ecotoxicology, Research Center for Eco-Environmental Sciences, Chinese Academy of Sciences, P.0. Box 2871, \\ Beijing 100085, China \\ ${ }^{\mathrm{b}}$ Department of Chemistry, Tsinghua University, Beijing 100084, China \\ c Provincial Key Laboratory of Research on Wastewater Treatment Technology, Anhui Academy of Environmental Science Research, Hefei, 230061, China
}

\section{A R T I C L E I N F O}

\section{Article history:}

Received 10 September 2008

Received in revised form

25 November 2008

Accepted 24 December 2008

Available online 31 December 2008

\section{Keywords:}

Molecularly imprinted polymer

2,4,6-Trichlorophenol

Phenolic compounds

Molecularly imprinted solid-phase

extraction

High performance liquid chromatography

\begin{abstract}
A B S T R A C T
The molecularly imprinted bulk polymer with 2,4,6-trichlorophenol (2,4,6-TCP) as the template molecule and methylacrylic acid (MAA), ethylene glycol dimethacrylate (EGDMA) as functional monomer and the crosslinker, respectively, has been prepared and applied to the molecularly imprinted solid-phase extraction (MISPE) procedure for selective preconcentration of phenolic compounds from environmental water samples. Various parameters affecting the extraction efficiency of the polymer have been evaluated to optimize the selective preconcentration of the phenolic compounds from aqueous samples. The characteristics of the MISPE method were valided by HPLC. The recoveries ranged between $90 \%$ and $98 \%$ (RSD: $0.9-2.3 \%, n=3$ ) for tap water, between $85 \%$ and $105 \%$ (RSD: $2.6-4.9 \%, n=3$ ) for river water, between $78 \%$ and $98 \%$ (RSD: $2.6-5.4 \%, n=3$ ) for sewage water fortified with $0.4 \mathrm{mg} \mathrm{L}^{-1}$ of phenol, 4-chlorophenol (4-CP), 2,4-dichlorophenol (2,4-DCP), pentachlorophenol (PCP). It was demonstrated that this MISPEHPLC method could be applied to direct preconcentration and determination of phenolic compounds in environmental water samples.
\end{abstract}

Crown Copyright (C) 2009 Published by Elsevier B.V. All rights reserved.

\section{Introduction}

Water quality control is one of the most critical issues in environmental analytical chemistry today. Phenolic compounds can be released into the environment directly and indirectly, such as industrial effluents, conversion products from natural and synthetic chemicals, including pesticides [1]. In recent years, phenolic compounds have been detected in surface water and groundwater in both Asia and Europe. Incomplete removal of these compounds in municipal sewage treatment plants (STP) is considered to be one of the main sources of phenolic compounds entrance into the environment.

One of the main interests in the determination of phenolic compounds in the environment arises from the fact that they are considered as priority pollutants since they are harmful to organ-

\footnotetext{
* Corresponding authors at: State Key Laboratory of Environmental Chemistry and Ecotoxicology, Research Center for Eco-Environmental Sciences, Chinese Academy of Sciences, P.O. Box 2871, Beijing 100085, China. Tel.: +86 1062841953 ; fax: +861062841953.

E-mail addresses: zlx@rcees.ac.cn (L.-X. Zhao),jmlin@mail.tsinghua.edu.cn (J.-M. Lin).
}

isms even at low concentrations and many of them have been classified as hazardous pollutants because of their potential to harm human health $[1,2]$. Especially for chlorophenols (CPs), which are a group of phenolic compounds, are listed in the US EPA's Clean Water Act [3] and European Decision 2455/2001/EC [4] for their highly toxic and barely biodegradable properties. The world market for CPs is fairly stable at ca. $100 \mathrm{kt}$ per year [4]. In China, CPs are more frequently detected at higher concentrations in the rivers of North China compared with those of South China and CPs contamination in the surface water of China is similar to other places of the world [5]. Therefore, it appears of great interest to design efficient and sensitive analytical methods which are able to detect trace-level of chlorophenols from the environmental waste water.

Analytical techniques used in determination of phenolic compounds are mainly high performance liquid chromatography (HPLC) [1], particularly reversed-phase liquid chromatography (RPLC), and also capillary electrophoresis (CE) [6,7] in combination with ultraviolet (UV) detection, or mass spectrometry (MS) [8]. Moreover, gas chromatography (GC) with flame ionization detection (FID), electron-capture detection and MS are common tools for the analysis of phenols, usually after derivatization $[9,10]$. To achieve the necessary levels of sensitivity, an enrichment step is needed before chromatographic analysis [1]. Solid-phase extrac- 
tion (SPE), nowadays, is a well established technique and has been used for the preconcentration and cleaning up of numerous different classes of compounds in a variety of matrices [11-13]. Due to the popularity of SPE, many new functionalized polymeric sorbents and highly cross-linked polymers have recently appeared as alternatives to conventional SPE materials in order to facilitate the trace enrichment of polar analytes. Moreover, there has been a lot of recent interest in the development of selective SPE sorbents that yield cleaner extracts in the analysis of complex matrixes, e.g., blood, urine, and environmental water samples. On the other hand, most of these sorbents does not show high selectivity for specific analytes, thus allowing the extraction of possible concomitants. Therefore, a separation methodology with built-in selectivity for the target compounds is urgently required. An elegant alternative for solving/minimizing the drawback aforementioned is the use of sorbents with specific binding sites for the single molecule, as molecularly imprinted polymers.

Molecularly imprinted polymers (MIP) are man-made polymers with a predetermined selectivity toward a given analyte or a group of structurally related species [14,15]. These materials have been employed in fields where a certain degree of selectivity is required such as chromatography [11,16-18], sensors [19,20], immunoassays $[21,22]$ and catalysts $[23,24]$. The most significant advantages of MIPs, as compared to biological receptors, such as antibodies, enzymes, nucleic acids, or cells, include their mechanical and chemical stability, high selectivity, low cost of preparation, and wide range of operating conditions $[25,26]$. The use of MIPs as selective sorbent materials allows performing a customized sample treatment step prior to the final determination. This is of special interest when the sample is complex and the presence of interferences could prevent final quantification by typical chromatographic techniques coupled to common detectors. Coupling MIPs with SPE is possible to combine the advantages of both molecular recognition and traditional separation methods. Thus, molecularly imprinted solidphase extraction (MISPE) presents the high specificity, selectivity and sensitivity of the molecular recognition mechanism and the high resolving power of separation methods [27]. Due to these advantages provided by MISPE, past years have seen a growing interest in this area and it has been extensively reviewed [28-30].

Recently, several papers have been published describing the application molecular imprinted polymers of halogenated phenols as solid-phase extraction materials, and a certain degree of selectivity has been shown [31-34]. To the best of our knowledge, 2,4,6-trichlorophenol (2,4,6-TCP) molecularly imprinted polymers were not prepared and used as the SPE sorbents to eliminate the phenolic compounds from environmental samples. In this work, 2,4,6-TCP molecularly imprinted polymers were prepared through bulk polymerization method using 2,4,6-TCP as template molecule, MAA as the functional monomer and EGDMA as the cross linking agent. The extraction efficiency of phenolic compounds from environmental water samples using an MISPE method coupled with HPLC was described. Analytical parameters of the method, i.e. linearity, detection limits and repeatability were established at low $\mu \mathrm{g} \mathrm{L}^{-1}$ level of concentration and quantitative aspects in sewage and river water were studied. The method had been validated and applied to determine phenolic compounds from environmental water with satisfactory results.

\section{Experiment}

\subsection{Reagents and apparatus}

The chemicals for the polymer syntheses (2,4,6-TCP, methyacrylic acid (MAA), ethylene glycol dimethacrylate (EGDMA)) and phenol, 4-chlorophenol (4-CP), 2,4-dichlorophenol (2,4-DCP), pentachlorophenol (PCP) were all from Acros Organics, 2,2'- azobisisobutyronitrile (AIBN) from Shanghai No. 4 Reagent \& H.V. Chemical Co. Ltd. (Shanghai China), and acetonitrile from J.T. Baker (USA). The monomers were purified prior to use in order to remove stabilizers. The AIBN was recrystallized from ethanol. Acetic acid was purchased from Beijing Chemical Reagent Company (Beijing, China), methanol, ethanol and toluene were all from Beijing Bei Hua Fine Chemical SCO, Ltd. (Beijing, China).

The chemicals for HPLC were at least of HPLC grade. Stock standard solutions of phenol, 4-CP, 2,4-DCP, 2,4,6-TCP, PCP were fresh prepared in acetonitrile, and stored at $4{ }^{\circ} \mathrm{C}$ in a refrigerator. All working solutions were prepared by diluting the stock standard solutions with deionized ultrapure water $\left(18.3 \mathrm{M} \Omega \mathrm{cm}^{-1}\right.$, EasyPure $^{\mathrm{TM}}$ LF Barnstead, USA) to the needed concentrations correspondingly.

The resulting bulk molecularly imprinted polymers were characterized by scanning electron microscope (SEM) (S-3000N, Hitachi, Japan) and by nitrogen adsorption/desorption analysis of Brunauer-Emmett-Teller (ASAP 2000, Micromeritics, USA).

To evaluate the polymers, ground polymer particles were suspended in water, the solutions were determined with the UV2401PC spectrophotometer (Shimadzu Corporation, Japan). The chromatographic evaluation of the polymers was also carried out: ground polymer particles were suspended in ethanol by sonication and then slurry packed into $5 \mathrm{~cm} \times 0.46 \mathrm{~cm}$ i.d. stainless steel HPLC columns at 2500 psi using an air-driven fluid pump (6752B-100, Beijing, China) with ethanol as the solvent.

\subsection{Preparation of molecular imprinted polymers with bulk methods}

For the preparation of the 2,4,6-TCP imprinted polymer, the template molecule $2,4,6$-TCP $(1 \mathrm{mmol})$ was dissolved in acetonitrile $(6 \mathrm{~mL})$ in a $25-\mathrm{mL}$ thick-walled glass tube, the functional monomer MAA ( $4 \mathrm{mmol})$, the cross linking agent EGDMA ( $20 \mathrm{mmol})$ and the initiator AIBN $(0.66 \mathrm{mmol})$ were then added to above solution. The solution was purged with oxygen-free nitrogen for $10 \mathrm{~min}$, the glass tube was sealed under the nitrogen and then placed in a water bath at $60^{\circ} \mathrm{C}$. The polymerization was allowed to proceed for $24 \mathrm{~h}$. The resulting hard polymers were crushed, ground and sieved to obtain about 32-40 $\mu \mathrm{m}$ particles suitable for the MISPE evaluations. The nonimprinted polymer (NIP) was prepared without $2,4,6$-TCP as reference polymer based on the same procedure. MIP particles were washed with the mixture solution of acrtonitrile/acetic acid (90:10, $\mathrm{v} / \mathrm{v}$ ) using soxhlet extraction to remove the template molecules. Finally, the particles were dried in vacuum at $35^{\circ} \mathrm{C}$.

\subsection{Binding characteristic assays}

The binding properties of MIP to 2,4,6-TCP were studied by batch method as it were reported before [35]. In a typical rebinding experiment, $5.0 \mathrm{mg}$ washed and dried MIPs were added into $5 \mathrm{~mL}$ 2,4,6TCP at different concentrations. Then the suspensions were sealed and oscillated for $20 \mathrm{~h}$ at room temperature to ensure equilibration. After centrifugation at $3500 \mathrm{rpm}$ for $10 \mathrm{~min}$, the concentration of free 2,4,6-TCP in the supernatant was detected by measuring the absorbance with UV-2401PC spectrophotometer. The amount of $2,4,6$-TCP bound to the polymer was calculated by subtracting the concentration of free $2,4,6-\mathrm{TCP}$ from the initial concentration.

\subsection{The preparation of the MISPE and chromatographic conditions}

$300 \mathrm{mg}$ MIP particles were packed into an empty column (frit: $20 \mu \mathrm{m}$ ) which was served as a molecularly imprinted solid-phase extraction (MISPE). The MISPE cartridges were conditioned with $10 \mathrm{~mL}$ methanol and $10 \mathrm{~mL}$ water successively. Water sample was 
passed through the cartridge at $1.5-2.0 \mathrm{~mL} \mathrm{~min}^{-1}$ using a suction system. After the extracts were cleaned up by $2 \mathrm{~mL}$ of methanol, 25 min dryness of the cartridge was operated by vacuum to avoid incomplete fraction. Then the extracts were eluted with a solution of acetonitrile/acetic acid (acetonitrile:acetic acid =99.0:1.0, v/v). And finally the obtained extract solution was blown down under nitrogen to final volume of $0.5 \mathrm{~mL}$ for subsequent HPLC analysis.

High performance liquid chromatography (Shimadzu, Japan) for determining phenolic compounds consisted of a binary LC-10ADvp pump, an DGU-12A on-line degasser, a CTO-10Avp column oven, a SPD-10Avp UV-vis detector and SCL-10Avp system controller. The injection loop volume was $20 \mu \mathrm{L}$, and the analytical column was a $25 \mathrm{~cm} \times 0.46 \mathrm{~cm}$ i.d. spherical ODS. The mobile phase consisted of Milli-Q-quality water, as solvent $A$ and acetonitrile (containing $0.3 \%, v / v$, acetic acid) as solvent B. Both solvent A and solvent B were mixed prior to reaching the analytical column. The flow rate of the mobile phase was $0.5 \mathrm{~mL} \mathrm{~min}^{-1}$, and the gradient profile was $20-40 \% \mathrm{~B}$ from 0 to $5 \mathrm{~min}, 60 \% \mathrm{~B}$ at $7.5 \mathrm{~min}, 80 \% \mathrm{~B}$ at $20 \mathrm{~min}$, and then isocratic elution for $10 \mathrm{~min}$. The oven temperature was set at $40^{\circ} \mathrm{C}$ and compounds were detected at $294 \mathrm{~nm}$, except for phenol and 2,4-DCP which were detected at $280 \mathrm{~nm}$.

\subsection{Sample preparation}

Water samples were collected in glass bottles. After the essential filtration treatment, the collected samples were stored in dark at $4{ }^{\circ} \mathrm{C}$ and the further SPE procedure was accomplished within $24 \mathrm{~h}$ to avoid any microbial degradation of analytes.

Tap water samples were directly collected from the water faucet in laboratory. River water samples were obtained from Qinghe River in the northwest of Beijing. The raw sewage samples were collected from influent of sewage treatment plan (STP) which mainly receives domestic water from the uptown (population of $\sim 8000$ ) located around the laboratory.

Filtration was an essential step for real water samples to avoid blocking of SPE cartridges, even for the clean water such as tap water. In this study, freshly collected river water sample and tap water sample were filtered with $0.45 \mu \mathrm{m}$ filter while the sewage water was filtered with $0.20 \mu \mathrm{m}$ filter to remove suspended particles prior to extraction.

\section{Results and discussion}

\subsection{Polymer synthesis and characterization}

The majority of the work in the molecular imprinting field had relied on production of blocks of the imprinted polymer in some type of mould (usually a glass tube). The block of polymer

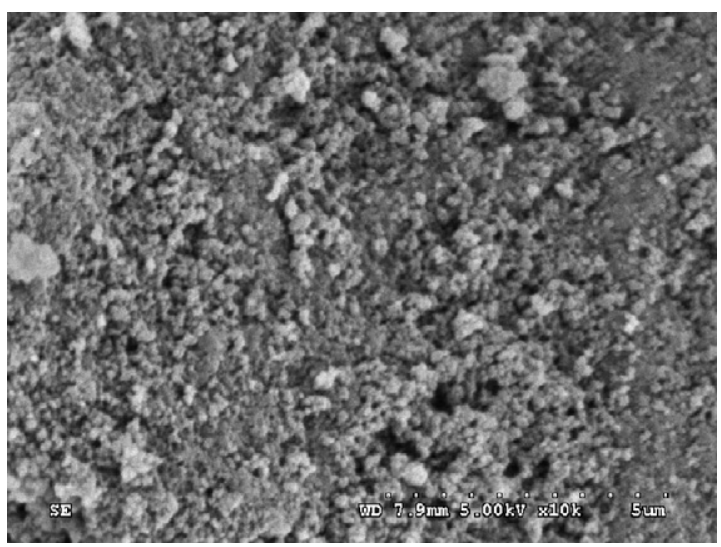

Fig. 1. The scanning electron micrographs (SEM) of the surface of the bulk polymer.
Table 1

Porosities of polymers determined by BET analysis.

\begin{tabular}{lrr}
\hline Sample & \multicolumn{1}{c}{ MIP } & \multicolumn{1}{c}{ NIP } \\
\hline Pore volume $\left(\mathrm{cm}^{3} \mathrm{~g}^{-1}\right)$ & 0.75 & 0.62 \\
BET surface area $\left(\mathrm{m}^{2} \mathrm{~g}^{-1}\right)$ & 287.15 & 256.04 \\
Pore size $(\AA)$ & 89.38 & 96.08 \\
\hline
\end{tabular}

was subsequently crushed, ground and sieved, either manually or mechanically, before being used for evaluation of the imprinted receptors obtained. This bulk polymer method was simple, fast and the obtained polymer could be used as the sorbent for SPE easily. Fig. 1 showed that the surface of the polymer was dominated by a mesoporous structure. Nitrogen adsorption/desorption analysis of Brunauer-Emmett-Teller was used to evaluate the pore volume and specific surface area of the polymer which were shown in Table 1. It was clearly evident that the polymer had large BET surface area and pore volume. This also showed that NIP and MIP particles were not significantly different in these aspects. Therefore, any differential 2,4,6-TCP loadings displayed by the polymer was not caused by the morphological distinction but by the imprinting effect.

\subsection{Evaluated the recognition efficiency of the bulk imprinted polymer}

The binding assays were carried out in aqueous solution instead of acetonitrile since the determination of 2,4,6-TCP was applied in water samples. $5.0 \mathrm{mg}$ washed and dried MIPs were added into $5 \mathrm{~mL} 2,4,6$-TCP at different concentrations. Then the suspensions were oscillated for $20 \mathrm{~h}$ at room temperature to ensure equilibration. After centrifugation at $3500 \mathrm{rpm}$, the concentration of free 2,4,6-TCP in the supernatant was detected with UV-2401PC spectrophotometer. The amount of 2,4,6-TCP bound to the polymer was calculated by subtracting the concentration of free $2,4,6-\mathrm{TCP}$ from the initial concentration. The data obtained from batch-type method with 2,4,6-TCP concentration from 10 to $350 \mathrm{mg} \mathrm{L}^{-1}$ were plotted according to the binding isotherm and shown in Fig. 2. The maximum amount $Q_{\max }$ was estimated to be $197.27 \mathrm{mg} \mathrm{g}^{-1}$ and $111.48 \mathrm{mg} \mathrm{g}^{-1}$ for MIP and NIP, respectively. The static adsorption capacity of the 2,4,6-TCP imprinted polymers was about two times of non-imprinted polymers. The results showed that the 2,4,6-TCP imprinted polymers had a higher adsorption capacity for $2,4,6$-TCP. They would be better to enrich trace $2,4,6$-TCP in the samples.

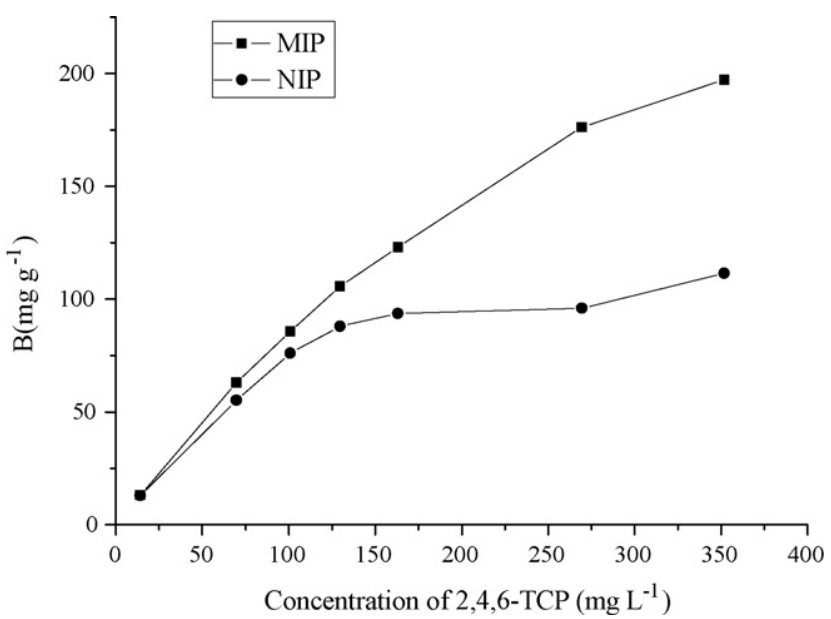

Fig. 2. Adsorption equilibrium isotherms of 2,4,6-TCP on the MIP and NIP at room temperature. 
Table 2

Capacity factor $\left(k^{\prime}\right)$ and normalized retention indices (RI) obtained from HPLC evaluation of the polymers.

\begin{tabular}{llll}
\hline & $k^{\prime}(\mathrm{MIP})$ & $k^{\prime}(\mathrm{NIP})$ & $\mathrm{RI}$ \\
\hline Phenol & 0.41 & 0.35 & 0.70 \\
$4-\mathrm{CP}$ & 0.74 & 0.62 & 0.71 \\
$2,4,6-\mathrm{TCP}$ & 1.90 & 1.13 & 1.00 \\
PCP & 3.14 & 2.57 & 0.73 \\
\hline
\end{tabular}

\subsection{Chromatographic evaluation of the polymers}

The columns packed with the polymers were washed with acetonitrile/acetic acid $(99.0 / 1.0, \mathrm{v} / \mathrm{v})$, to eliminate interfering compounds from the synthetic procedure, until a stable baseline was obtained. They were then evaluated under the chromatographic conditions described in Section 2 by injecting $0.4 \mathrm{mg} \mathrm{L}^{-1}$ solutions of phenol, 4-CP, 2,4,6-TCP and PCP as test analytes onto the columns. These phenolic compounds had different retention times, and for this reason, the data obtained in these analyses were normalized by calculating the normalized retention index (RI). The RI gave a measure of the degree of recognition for a given analyte. The template molecule gave a value of 1 by definition, and other, less strongly retained, compounds give smaller values. The RI was defined as Eq. (1).

$\mathrm{RI}=\frac{k_{\text {analyte }}^{\prime}(\mathrm{MIP}) / k_{\text {analyte }}^{\prime}(\mathrm{NIP})}{k_{\text {template }}^{\prime}(\mathrm{MIP}) / k_{\text {template }}^{\prime}(\mathrm{NIP})}$

where $k^{\prime}$ is the capacity factor. To calculate $k^{\prime}$, the dead time of the MIP and the control columns was measured by injecting acetone as a void marker. Table 2 showed the $k^{\prime}$ value and the corresponding RI value for the test phenolic compounds in both columns. PCP, 4-CP and $\mathrm{Ph}$ had smaller RI values than 2,4,6-TCP, and this result verifies the imprinting effect.

\subsection{Optimization of the MISPE procedure}

A preconcentration process was indispensable due to the relative low amount of the analytes in the environmental water. SPE, as the most effective method for the extraction of most organic compounds, was employed for the preconcentration of the analytes. The polymers in the MISPE were initially washed with solvent (acetonitrile containing $1 \%, v / v$, acetic acid) to remove any residual template that could potentially interfere with the analysis, until 2,4,6-TCP was no longer detectable in the eluate. The MISPE process was optimized by evaluating the washing solvent, the composition and volume of the eluting solvent and the flow rate of the loading solution.

\subsubsection{Washing solvent optimization}

The kind of the washing solution plays a vital role on the selectivity of the MIPs and thus a careful optimization is needed. $10 \mathrm{~mL}$ samples containing $0.4 \mathrm{mg} \mathrm{L}^{-1} 2,4,6$-TCP dissolved in water, were loaded onto the cartridge, after washed with $2 \mathrm{~mL}$ of methanol or acetonitrile or the mixture solution of methanol:acetonitrile $(1: 1$, $\mathrm{v} / \mathrm{v}$ ), and eluted with $2 \mathrm{~mL}$ of acetonitrile (containing $1 \%, \mathrm{v} / \mathrm{v}$, acetic acid). The concentration of the 2,4,6-TCP was determined with HPLC.

Fig. 3 showed the effect of the washing solution on the recovery of 2,4,6-TCP when $10 \mathrm{~mL}$ sample was loaded into the MIP cartridge. An average extraction recovery of $90 \%$ was obtained with $2 \mathrm{~mL}$ methanol. However, the average extraction recovery was only $72 \%$ or $63 \%$ when $2 \mathrm{~mL}$ of the mixture solution of methanol:acetonitrile $(1: 1, v / v)$ or acetonitrile was used as the washing solution. Therefore, in the later experiment, $2 \mathrm{~mL}$ methanol as used as the washing solution.

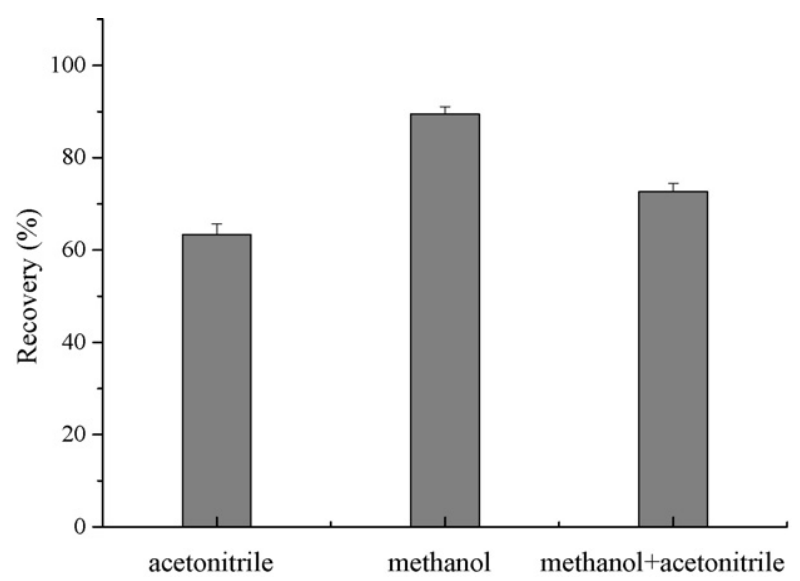

Fig. 3. The recovery of washing solution for MISPE experiment.

\subsubsection{Elution solvent optimization}

$10 \mathrm{~mL}$ samples containing $0.4 \mathrm{mg} \mathrm{L}^{-1} 2,4,6$-TCP dissolved in water, were loaded onto the cartridge, after washed with $2 \mathrm{~mL}$ of methanol, eluted with $2 \mathrm{~mL}$ of acetonitrile, solvent A (acetonitrile containing $1 \%, \mathrm{v} / \mathrm{v}$, acetic acid), solvent $\mathrm{B}$ (acetonitrile containing
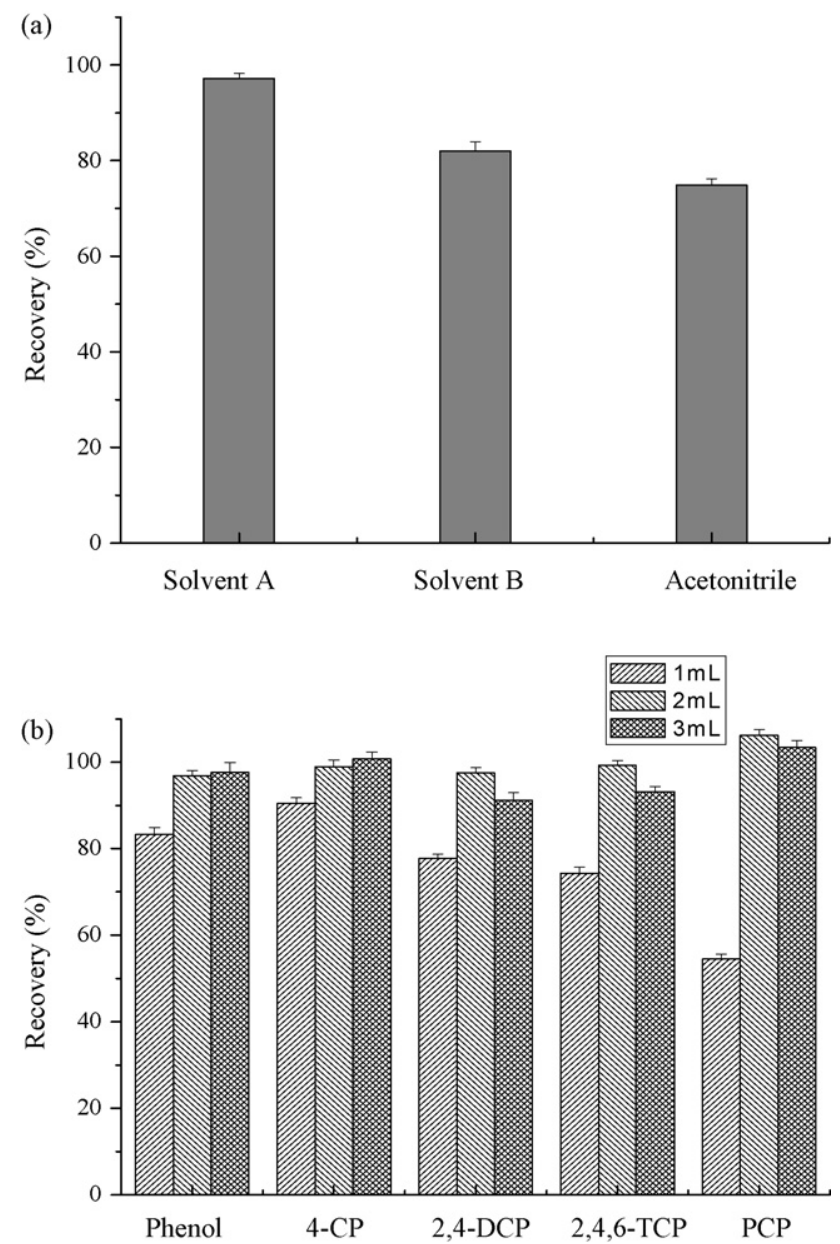

Fig. 4. The recovery for MISPE experiment. (a) Extraction the recovery of different elution solvent for 2,4,6-TCP, solvent A (acetonitrile containing $1 \%, \mathrm{v} / \mathrm{v}$, acetic acid), solvent B (acetonitrile containing $10 \%, \mathrm{v} / \mathrm{v}$, acetic acid). (b) Extraction recoveries (\%) obtained on the MIP for five phenolic compounds spiked with $1.0 \mathrm{mg} \mathrm{L}^{-1}$ of each compound using a washing step with different amount $(1 \mathrm{~mL}, 2 \mathrm{~mL}, 3 \mathrm{~mL})$ of solvent (acetonitrile containing $1 \%, \mathrm{v} / \mathrm{v}$, acetic acid). (1) Phenol, (2) 4-CP, (3) 2,4-DCP, (4) 2,4,6-TCP, (5) PCP. 


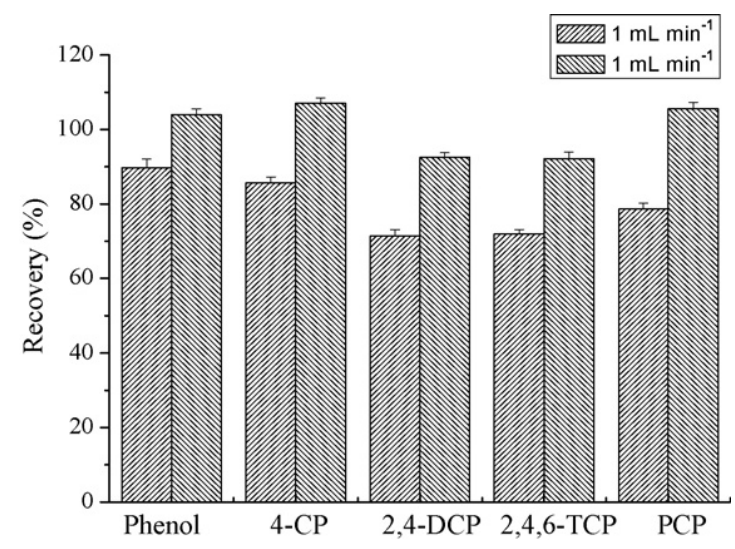

Fig. 5. Extraction recoveries (\%) obtained on the MIP for five phenolic compounds spiked with $1.0 \mathrm{mg} / \mathrm{L}$ of each compound using a washing step with differentflow rate $\left(1 \mathrm{~mL} \mathrm{~min}^{-1}, 2 \mathrm{~mL} \mathrm{~min}^{-1}\right.$ ) of solvent (acetonitrile containing $1 \%, \mathrm{v} / \mathrm{v}$, acetic acid). (1) Phenol, (2) 4-CP, (3) 2,4-DCP, (4) 2,4,6-TCP, (5) PCP.

$10 \%, v / v$, acetic acid). The concentration of the $2,4,6-\mathrm{TCP}$ was determined with HPLC.

To confirm that the acetic acid in the elution solvent (solvent A and B) played an important role in desorbing 2,4,6-TCP from the MIP, pure acetonitrile was tested as the elution solvent. Under these conditions, the 2,4,6-TCP recovery decreased from 97 to $75 \%$. Thus, the addition of acetic acid results in an increase in the recovery of 2,4,6-TCP. The most likely explanation was that acetic acid competed with 2,4,6-TCP for the functional groups in the binding sites, but too many acetic acid did not benefit the eluting. From Fig. 4a, we could come to the conclusion that solvent A (acetonitrile containing $1 \%, v / v$, acetic acid) was the optimal solvent for the following study.

To determine the optimum eluting volume, a $10 \mathrm{~mL}$ water sample spiked with $0.4 \mathrm{mg}$ of phenol, 4-CP, 2,4,-DCP, 2,4,6-TCP and PCP, was percolated through the MIP, and different volumes (1.0, 2.0 or $3.0 \mathrm{~mL}$ ) of a mixture of solvent A (acetonitrile containing $1 \%$, $\mathrm{v} / \mathrm{v}$, acetic acid) were applied in the washing step, and the eluates were analyzed by HPLC. The results are shown in Fig. $4 \mathrm{~b}$. The use of $1.0 \mathrm{~mL}$ of eluting solvent allowed recoveries lower than 91\% (RSD 4.5-6.3\%, $n=3$ ) in the MIP cartridge for all the phenolic compounds tested, especially for PCP, whose recovery was only $54 \%$. However, the use of $2.0 \mathrm{~mL}$ of eluting solvent allowed recoveries from $97 \%$ to $106 \%$ (RSD $4.5-6.3 \%, n=3$ ), which were very acceptable for MISPE.

When a eluting volume of $3.0 \mathrm{~mL}$ was applied instead, the recoveries were about $91-103 \%$, which did not improve much more than that of a eluting volume of $2.0 \mathrm{~mL}$. In conclusion, a volume of $2 \mathrm{~mL}$ of solvent A (acetonitrile containing $1 \%, v / v$, acetic acid) was selected for the eluting step.

\subsubsection{Effect of the flow rate of the loading solution}

Fig. 5 showed the effect of the flow rate of the loading solution on the recovery of phenolic compounds when $10 \mathrm{~mL}$ of a solution of the compounds was loaded into the MIP cartridge. An aver-

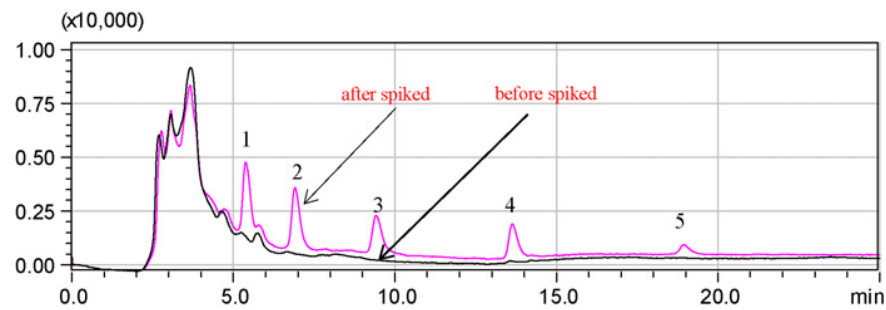

Fig. 6. Chromatograms of sewage water sample before (black line) and after (red line) spiked at $0.4 \mathrm{mg} \mathrm{L}^{-1}$ of each analyte. (1) Phenol, (2) 4-CP, (3) 2,4-DCP, (4) 2,4,6TCP, (5) PCP.

age extraction recovery of $92-106 \%$ was obtained at flow rate of $2.0 \mathrm{~mL} \mathrm{~min}^{-1}$. However, higher rates led to a continuous decrease in the recovery values as the interaction time between the analytes and the sorbent was decreased, and the use of lower rates led to a continuous decrease in the recovery values as the interaction time between the analytes and the sorbent was extended. The loading rate of $2.0 \mathrm{~mL} \mathrm{~min}^{-1}$ was selected for further experiments.

\subsubsection{Experiment of the sample volume}

The effect of sample volume (from 2 to $15 \mathrm{~mL}, 0.10 \mathrm{mg} / \mathrm{L}$ ) on extraction efficiency was investigated. When the sample volume were $2,4,6,8,10,15 \mathrm{~mL}$, the recoveries were $95 \%, 96 \%, 94 \%, 93 \%$, $93 \%, 64 \%$, respectively. The acceptable recovery was observed when sample volumes were increased to $10 \mathrm{~mL}$, it seemed to be the tolerated volume for breakthrough. Hence, $10 \mathrm{~mL}$ was selected as the optimal sample volume.

\subsection{Determination of phenolic compounds with HPLC}

Determination of phenolic compounds (phenol, 4-CP, 2,4-DCP, 2,4,6-TCP and PCP) with HPLC was carried out as described in Section 2.4. The mobile phase consisted of water, as solvent $A$ and acetonitrile (containing $0.3 \%, \mathrm{v} / \mathrm{v}$, acetic acid) as solvent $\mathrm{B}$. The flow rate of the mobile phase was $0.5 \mathrm{~mL} \mathrm{~min}^{-1}$. The oven temperature was set at $40 \mathrm{C}$, the injection volume was $20 \mu \mathrm{L}$, and all compounds were detected at $294 \mathrm{~nm}$, except for phenol and 4-CP which were detected at $280 \mathrm{~nm}$.

The method performance was evaluated by the determination of the linearity, sensitivity, repeatability, and accuracy of the method.

The linearity of the calibration curves were obtained by the determination of the peak areas from analysis of 0.05 to $2.0 \mathrm{mg} \mathrm{L}^{-1}$ of each analyte and the all $r$-values were higher than 0.996 (in Table 3).

The limits of detection (LOD), defined as the lowest analyte concentration with a signal-to-noise ratio of 3 , were also investigated through the detection of spiked Mili-Q water at serial concentrations. The results showed that the LODs fell between 0.57 and $1.08 \mu \mathrm{g} \mathrm{L}{ }^{-1}$, which indicated this method could be used to detect some polluted water samples.

Table 3

The characteristic of the standard curve.

\begin{tabular}{|c|c|c|c|c|c|c|}
\hline Phenolic compounds & Wavelength (nm) & $a$ & $b$ & $R$ & Linear scope $\left(\mathrm{mg} \mathrm{L}^{-1}\right)$ & $\operatorname{LOD}\left(\mu \mathrm{g} \mathrm{L}^{-1}\right)$ \\
\hline Phenol & 280 & 304.93 & 42226 & 0.9965 & $0.05-20.0$ & 0.79 \\
\hline 4-CP & 280 & -1.829 & 38343 & 0.9982 & $0.05-20.0$ & 1.08 \\
\hline 2,4-DCP & 294 & -224.8 & 31442 & 0.9999 & $0.05-20.0$ & 0.76 \\
\hline $2,4,6-\mathrm{TCP}$ & 294 & -412.62 & 33510 & 0.9998 & $0.05-20.0$ & 0.57 \\
\hline PCP & 294 & 121.55 & 21481 & 0.9978 & $0.05-20.0$ & 0.70 \\
\hline
\end{tabular}

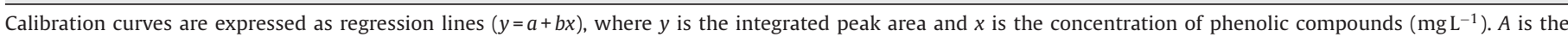
intercept, $b$ is the slope and $R$ is the relative coefficient, LOD is limit of detection at 3:1 signal-to-noise ratio. 
Table 4

The limit of detection, accuracy and precision in tap water, river water and raw sewage matrixes.

\begin{tabular}{|c|c|c|c|c|c|c|c|c|c|c|c|c|c|c|c|}
\hline & \multicolumn{5}{|l|}{ Tap water } & \multicolumn{5}{|l|}{ River water } & \multicolumn{5}{|l|}{ Raw sewage } \\
\hline & \multicolumn{3}{|l|}{ MIP } & \multicolumn{2}{|l|}{ NIP } & \multicolumn{3}{|l|}{ MIP } & \multicolumn{2}{|l|}{ NIP } & \multicolumn{3}{|l|}{ MIP } & \multicolumn{2}{|l|}{ NIP } \\
\hline & $\mathrm{LOD}\left(\mathrm{mg} \mathrm{L}^{-1}\right)$ & $R^{\mathrm{a}}(\%)$ & $\mathrm{RSD}^{\mathrm{b}}(\%)$ & $R^{\mathrm{a}}(\%)$ & $\mathrm{RSD}^{\mathrm{b}}(\%)$ & $\mathrm{LOD}\left(\mathrm{mg} \mathrm{L}^{-1}\right)$ & $R(\%)$ & RSD (\%) & $R^{\mathrm{a}}(\%)$ & $\operatorname{RSD}^{\mathrm{b}}(\%)$ & $\operatorname{LOD}\left(\mathrm{mg} \mathrm{L}^{-1}\right)$ & $R(\%)$ & RSD (\%) & $R^{\mathrm{a}}(\%)$ & $\operatorname{RSD}^{\mathrm{b}}(\%)$ \\
\hline Phenol & 0.016 & 97 & 1.9 & 64 & 2.3 & 0.033 & 86 & 4.9 & 58 & 4.7 & 0.050 & 84 & 2.7 & 55 & 3.7 \\
\hline 4-CP & 0.018 & 95 & 0.9 & 60 & 1.1 & 0.038 & 100 & 4.7 & 55 & 4.3 & 0.056 & 86 & 4.3 & 52 & 4.1 \\
\hline 2,4-DCP & 0.028 & 90 & 2.3 & 61 & 2.4 & 0.056 & 85 & 3.5 & 56 & 3.7 & 0.067 & 78 & 2.5 & 53 & 4.8 \\
\hline $2,4,6-\mathrm{TCP}$ & 0.045 & 90 & 2.2 & 67 & 1.1 & 0.048 & 87 & 4.8 & 60 & 4.1 & 0.059 & 81 & 3.9 & 56 & 3.6 \\
\hline PCP & 0.034 & 98 & 2.3 & 58 & 2.6 & 0.068 & 105 & 2.6 & 56 & 2.4 & 0.082 & 98 & 4.4 & 55 & 4.0 \\
\hline
\end{tabular}

${ }^{a} R$, Recovery for the evaluation of accuracy (spiked at $0.4 \mathrm{mg} \mathrm{L}^{-1}$ of each analyte in $10 \mathrm{~mL}$ water samples).

b RSD, Relative standard deviation for the evaluation of precision $(n=3)$.

\subsection{MISPE of real water sample}

The application of the imprinted polymer for MISPE of phenolic compounds in complex matrixes was investigated. The tap water, river water and sewage water were extracted using MISPE procedure described in Section 2.4. However, no target analytes were detected, it was probably because there was little phenolic compounds in Qinghe river in Beijing and the sewage water. Thus, to assess matrix effects, water samples were spiked and the extracted performance was evaluated. Fig. 6 showed the liquid chromatogram of the phenolic compounds in sewage water before and after spiking at $0.4 \mathrm{mg} \mathrm{L}^{-1}$. It indicted that the matrix effects on MISPE were negligible. Table 4 showed the LODs and recoveries of spiked water sample.

The recoveries obtained with MIPs as the solid-phase sorbent for phenol, 4-CP, 2,4-DCP, 2,4,6-TCP, PCP ranged between 90\% and 98\% for tap water, between $85 \%$ and $105 \%$ for river water and between $78 \%$ and $98 \%$ for sewage water at both fortification levels (Table 4 ). The RSD values of targets were lower than $5 \%$ which were also excellent. However, the recoveries obtained with NIP as the solid-phase sorbent for phenol, 4-CP, 2,4-DCP, 2,4,6-TCP, PCP were only between $58 \%$ and $67 \%$ for tap water, between $55 \%$ and $60 \%$ for river water and between $52 \%$ and $56 \%$ for sewage water at both fortification levels (Table 4). It demonstrated that 2,4,6-TCP MIPs had a satisfactory performance for the enrichment of phenolic compound. The extraction recovery in this report was also compared to the common LC, PLRP-S, porous graphitic carbon or GC methods [36-39] which used $\mathrm{C}_{18}$ or Carbon black as the sorbents and extraction recoveries were below $76 \%$, the results showed the extraction recovery was improved significantly in this report because molecularly imprinted polymers have more selectivity to the target compounds.

\section{Conclusions}

The 2,4,6-TCP imprinted polymer was prepared by bulk polymerization using MAA and EGDMA as the monomer and crosslinker, respectively. The obtained polymer showed good selectivity and enrichment efficiency. Thus, a method was successfully developed by using the MAA-co-EGDMA polymer as the MISPE coupled with HPLC for enrichment and analysis of phenolic compounds in environmental water. The high recoveries (78-105\%) and satisfied precision $(0.9-4.9 \%)$ for all the analytes proved that the method was valid for the analysis of some phenolic compounds mentioned above in different matrixes (tap water, river water, sewage water). From this point of view, the MISPE-HPLC method was developed to determine some phenolic compounds in environmental water directly and the results of the recovery validated the method.

\section{Acknowledgements}

The authors gratefully acknowledge financial support of the National Natural Science Foundation of China (Grant Nos.
20437020, 20575073 and 20621703), the Major Research Program of the Chinese Academy of Sciences (KZCX3-SW-432) and the Science and Technological Fund of Anhui Province for Outstanding Youth (08040106832).

\section{References}

[1] H. Bagheri, A. Mohammadi, A. Salemi, On-line trace enrichment of phenolic compounds from water using a pyrrole-based polymer as the solid-phase extraction sorbent coupled with high-performance liquid chromatography, Anal. Chim. Acta 513 (2004) 445-449.

[2] O. Hamdaoui, E. Naffrechoux, J. Hazar, Modeling of adsorption isotherms of phenol and chlorophenols onto granular activated carbon. Part I. Two-parameter models and equations allowing determination of thermodynamic parameters, J. Hazard. Mater. 147 (2007) 381-394.

[3] L.H. Keith, W.A. Telliard, Priority pollutrants, Environ. Sci. Technol. 13 (1979) 416-423.

[4] M. Abecassis-Wolfovich, M.V. Landau, A. Brenner, M. Herskowitz, Lowtemperature combustion of 2,4,6-trichlorophenol in catalytic wet oxidation with nanocasted Mn-Ce-oxide catalyst, J. Catal. 247 (2007) 201-213.

[5] J.J. Gao, L.H. Liu, X.R. Liu, H.D. Zhou, S.B. Huang, Z.J. Wang, Levels and spatial distribution of chlorophenols-2,4-dichlorophenol, 2,4,6-trichlorophenol, and pentachlorophenol in surface water of China, Chemosphere 71 (2008) 1181-1187.

[6] D. Puig, D. Barceló, Determination of phenolic compounds in water and waste water, Trac-Trend Anal. Chem. 15 (1996) 362-375.

[7] E. Blanco, M.C. Casais, M.C. Mejuto, R. Cela, Analysis of tetrabromobisphenol A and other phenolic compounds in water samples by non-aqueous capillary electrophoresis coupled to photodiode array ultraviolet detection, J. Chromatogr. A 1071 (2005) 205-211.

[8] O. Jáuregui, M.T. Galceran, Determination of phenols in water by on-line solid-phase disk extraction and liquid chromatography with electrochemical detection, Anal. Chem. Acta 340 (1997) 191-199.

[9] H. Bagheri, A. Mohammadi, Pyrrole-based conductive polymer as the solidphase extraction medium for the preconcentration of environmental pollutants in water samples followed by gas chromatography with flame ionization and mass spectrometry detection, J. Chromatogr. A 1015 (2003) 23-30.

[10] H. Faraji, $\beta$-Cyclodextrin-bonded silica particles as the solid-phase extraction medium for the determination of phenol compounds in water samples followed by gas chromatography with flame ionization and mass spectrometry detection, J. Chromatogr. A 1087 (2005) 283-288.

[11] N. Masque, R.M. Marce, F. Borrull, P.A.G. Cormack, D.C. Sherrington, Synthesis and evaluation of a molecularly imprinted polymer for selective on-line solidphase extraction of 4-nitrophenol from environmental water, Anal. Chem. 72 (2000) 4122-4126.

[12] E.C. Figueiredo, C.R. Teixeira Tarley, L.T. Kubota, S. Rath, M.A. Zezzi Arruda, On-line molecularly imprinted solid phase extraction for the selective spectrophotometric determination of catechol, Microchem. J. 85 (2007) 290-296.

[13] B. Bjarnason, L. Chimuka, O. RamstrÖm, On-line solid-phase extraction of triazine herbicides using a molecularly imprinted polymer for selective sample enrichment, Anal. Chem. 71 (1999) 2152-2156.

[14] C.H. Lu, W.H. Zhou, B. Han, H.H. Yang, X. Chen, X.R. Wang, Surface-imprinted core-shell nanoparticles for sorbent assays, Anal. Chem. 79 (2007) 54575461.

[15] G. Wulff, Enzyme-like catalysis by molecularly imprinted polymers, Chem. Rev. $102(2002) 1-27$

[16] E. Caro, R.M. Marce, P.A.G. Cormack, D.C. Sherrington, F. Borrull, On-line solidphase extraction with molecularly imprinted polymers to selectively extract substituted 4-chlorophenols and 4-nitrophenol from water, J. Chromatogr. A 995 (2003) 233-238.

[17] R. Garcia, C. Pinel, C. Madic, M. Lemaire, Ionic imprinting effect in gadolinium/lanthanum separation, Tetrahedron Lett. 39 (1998) 8651-8654.

[18] K. MÖller, U. Nilsson, C. Crescenzi, Synthesis and evaluation of molecularly imprinted polymers for extracting hydrolysis products of organophosphate flame retardants, J. Chromatogr. A 938 (2001) 121-130. 
[19] N. Tsuru, M. Kikuchi, H. Kawaguchi, S. Shiratori, A quartz crystal microbalance sensor coated with MIP for "Bisphenol A" and its properties, Thin Solid Films 449 (2006) 380-385.

[20] D.Y. He, ZJ. Zhang, H.J. Zhou, Y. Huang, Micro flow sensor on a chip for the determination of terbutaline in human serum based on chemiluminescence and a molecularly imprinted polymer, Talanta 69 (2006) 12151220.

[21] N. Lavignac, C.J. Allender, K.R. Brain, Current status of molecularly imprinted polymers as alternatives to antibodies in sorbent assays, Anal. Chim. Acta 510 (2004) 139-145.

[22] S.A. Piletsky, E.V. Piletska, A. Bossi, K. Karim, P. Lowe, A.P.F. Turner, Substitution of antibodies and receptors with molecularly imprinted polymers in enzyme-linked and fluorescent assays, Biosens. Bioelectron. 16 (2001) 701707.

[23] J.T. Huang, S.H. Zheng, J.Q. Zhang, Molecularly imprinting of polymeric nucleophilic catalysts containing 4-alkylaminopyridine functions, Polymer 45 (2004) 4349-4354.

[24] G.E. Southard, K.A. Van Houten, G.M. Murray, Soluble and processable phosphonate sensing star molecularly imprinted polymers, Macromolecules 40 (2007) 1395-1400.

[25] N.A. O'Connor, D.A. Paisner, D. Huryn, K.J. Shea, Screening of 5-HT 1 A receptor antagonists using molecularly imprinted polymers, J. Am. Chem. Soc. 129(2007) 1680-1689.

[26] K. Nemoto, T. Kubo, M. Nomachi, T. Sano, T. Matsumoto, K. Hosoya, T. Hattori, K. Kaya, Simple and effective 3D recognition of domoic acid using a molecularly imprinted polymer, J. Am. Chem. Soc. 129 (2007) 13626-13632.

[27] F. Puoci, M. Curcio, G. Cirillo, F. Iemma, U.G. Spizzirri, N. Picci, Molecularly imprinted solid-phase extraction for cholesterol determination in cheese products, Food Chem. 106 (2008) 836-842.

[28] F.G. Tamayo, E. Turiel, A. Martín-Esteban, Molecularly imprinted polymers for solid-phase extraction and solid-phase microextraction: recent developments and future trends, J. Chromatogr. A 1152 (2007) 32-40.

[29] X.M. Jiang, C.D. Zhao, N. Jiang, H.X. Zhang, M.C. Liu, Selective solid-phase extraction using molecular imprinted polymer for the analysis of diethylstilbestrol, Food Chem. 108 (2008) 1061-1067.
[30] A. Guerreiro, A. Soares, E. Piletska, B. Mattiasson, S. Piletsky, Preliminary evaluation of new polymer matrix for solid-phase extraction of nonylphenol from water samples, Anal. Chim. Acta 612 (2008) 99-104.

[31] D.M. Han, G.Z. Fang X.P. Yan, Preparation and evaluation of a molecularly imprinted sol-gel material for on-line solid-phase extraction coupled with high performance liquid chromatography for the determination of trace pentachlorophenol in water samples, J. Chromatogr. A 1100 (2005) 131-136.

[32] T. Kubo, K. Hosoya, Y. Watabe, T. Ikegami, N. Tanaka, T. Sano, K. Kaya, Polymerbased adsorption medium prepared using a fragment imprinting technique for homologues of chlorinated bisphenol A produced in the environment, J. Chromatogr. A 1029 (2004) 37-41.

[33] C. Baggiani, L. Anfossi, C. Giovannoli, C. Tozzi, Multivariate analysis of the selectivity for a pentachlorophenol-imprinted polymer, J. Chromatogr. B 804 (2004) 31- 41.

[34] C. Nicholls, K. Karim, S. Piletsky, S. Saini, S. Setford, Displacement imprinted polymer receptor analysis (DIPRA) for chlorophenolic contaminants in drinking water and packaging materials, Biosens. Bioelectron. 21 (2006) 1171- 1177.

[35] Y. Xiong, H.J. Zhou, Z.J. Zhang, D.Y. He, C. He, Flow-injection chemiluminescence sensor for determination of isoniazid in urine sample based on molecularly imprinted polymer, Spectrochim. Acta A 66 (2007) 341-346.

[36] R. Wissiack, E. Rosenberg, M. Grasserbauer, Comparison of different sorbent materials for on-line solid-phase extraction with liquid chromatographyatmospheric pressure chemical ionization mass spectrometry of phenols, J. Chromatogr. A 896 (2000) 159-170.

[37] E. Pocurull, M. Calull, R.M. Marcé, F. Borrull, Determination of phenolic compounds at low $\mu \mathrm{g} 1^{-1}$ levels by various solid-phase extractions followed by liquid chromatography and diode-array detection, J. Chromatogr. A 719 (1996) 105-112.

[38] D. Puig, D. Barceló, Comparison of different sorbent materials for on-line liquid-solid extraction followed by liquid chromatographic determination of priority phenolic compounds in environmental waters, J. Chromatogr. A 733 (1996) 371-381.

[39] H. Bagheri, M. Saraji, New polymeric sorbent for the solid-phase extraction of chlorophenols from water samples followed by gas chromatography-electroncapture detection, J. Chromatogr. A 910 (2001) 87-93. 